\title{
Observation of cyclotron resonance in the photoconductivity of two- dimensional electrons
}

\author{
J. C. Maan, Th. Englert, and D. C. Tsui a) \\ Max-Planck-Institut für Festkörperforschung, Hochfeld-Magnetlabor, 166X, F-38042 Grenoble, France
}

A. C. Gossard

Bell Laboratories, Murray Hill, New Jersey 07974

(Received 22 October 1981; accepted for publication 8 January 1982)

We have observed cyclotron resonance in the photoresponse of the two-dimensional electron gas at the interface of a GaAs/AlGaAs heterojunction. A $0.5 \%$ change in resistivity was obtained under resonance conditions for a far-infrared intensity of about $10^{-4} \mathrm{~W} / \mathrm{cm}^{2}$.

PACS numbers: 85.30.De, 85.60.Dw

Photoconductivity of two-dimensional (2D) electrons in surface inversion layers was first reported by Katayama $e t$ $a l .{ }^{1}$ It has since been successfully used as a spectroscopic tool to study the subband energy structure of the inversion layers. ${ }^{2}$ Cyclotron resonance (CR) in the photoconductivity of inversion layers on InSb and Si has recently been reported by Darr et $a l .^{3}$ and Lavine et al., ${ }^{4}$ respectively, after earlier unsuccessful attempts by Shiraki. ${ }^{5}$ However, simple, clear resonance was not observed in either experiment. The Shubnikov-de Haas $(\mathbf{S d H})$ oscillations are the dominant features in the data from these experiments and the $\mathrm{CR}$ only appears as a small modulation of the oscillation amplitude.

In this letter we report the observation of cyclotron resonance in the photoconductivity of the $2 \mathrm{D}$ electron gas in $\mathrm{GaAs}$. This is the first clear, resonancelike measurement of $\mathrm{CR}$ in the photoresponse of 2D electrons. The 2D electron gas is realized at a GaAs/AlGaAs heterojunction, which was grown by MBE (molecular beam epitaxy). Due to the conduction band discontinuity at the interface, electrons are transferred from the donors in the $\mathrm{AlGaAs}$ and confined to the GaAs at the GaAs-AlGaAs interface, forming a 2D electron gas. ${ }^{6}$ The high mobility, achieved by selectively doping only the AlGaAs, makes it possible the observation of a sharp CR response. The carrier density $n_{s}$ of our sample is sufficiently low that the period of the $\mathrm{SdH}$ oscillations is large enough to make the $C R$ signal well separated from the quantum oscillations. Both this fact and the high electron mobility made our sample much more favorable for observing a sharp resonance, which resembles more closely the $\mathrm{CR}$ line observed in absorption.

The sample used in the present experiment was a selectively doped $\mathrm{GaAs} / \mathrm{Al}_{x} \mathrm{Ga}_{1-x} \mathrm{As}(x=0.3)$ heterojunction with a low-temperature mobility of about $100000 \mathrm{~cm}^{2} / \mathrm{Vs}$ and a carrier density of $n_{s}=3.4 \times 10^{11} \mathrm{~cm}^{-2}$, corresponding to a Fermi energy $E_{F}=11.2 \mathrm{meV}$. The sample was immersed in liquid helium. The far-infrared radiation was generated by an optically pumped laser system, chopped and guided to the sample in the cryostat by light pipes. The magnetic field $B$ was produced with a $20-T$ Bitter coil. The sample was biased with a constant current of a few microamperes and the photoresponse signal was measured as a

"Also at Bell Laboratories, Murray Hill, New Jersey 07974.

Present address: Princeton University, Princeton, New Jersey 08544. change in the voltage drop across the sample using phasesensitive detection.

Figure 1 shows the photoresponse signal obtained with a far-infrared wavelength $\lambda=96 \mu \mathrm{m}$. It shows a sharp peak on an almost flat zero background. The resonance has a full linewidth at half-maximum of $\sim 0.3 \mathrm{~T}$. These data were taken at $4.2 \mathrm{~K}$, with a bias current $I=10 \mu \mathrm{A}$ and at a chopping frequency of $30 \mathrm{~Hz}$. The signal decreased by a factor of 5 when the chopping frequency was increased to $300 \mathrm{~Hz}$ but remained readily observable. A threefold signal increase was observed at $2 \mathrm{~K}$. The bias current dependence of the signal is shown in Fig. 2. At 30-Hz chopping frequency and a bias $I=10 \mu \mathrm{A}$ the signal corresponds to an increase in resistance of about $0.5 \%$ for $\lambda=96 \mu \mathrm{m}$ at an estimated radiation intensity of $100 \mu \mathrm{w} / \mathrm{cm}^{2}$. Similar but weaker resonances were observed for wavelengths $\lambda=170 \mu \mathrm{m}, 118.8 \mu \mathrm{m}$, and $70.6 \mu \mathrm{m}$. The inset in Fig. 1 shows the resonance position as determined from the photoresponse peaks for these different frequencies. The solid line indicates the CR position for an effective mass $m^{*}=0.070 m_{0}\left(m_{0}\right.$, free-electron mass), as



FIG. 1. Photoresponse as a function of the magnetic field for $\lambda=96 \mu \mathrm{m}$ at $4.2 \mathrm{~K}$ with bias $I=10 \mu \mathrm{A}$. The inset shows the resonance position and the solid line corresponds to $m^{*}=0.070 m_{0}$, as obtained from absorption measurements made on the same sample. 




FIG. 2. Photoresponse at CR with $\lambda=96 \mu \mathrm{m}$ as a function of bias current.

obtained from absorption measurements made on the same sample.

The additional structure around $15 \mathrm{~T}$ and the structures on the low field side of the resonance peak in Fig. 1 is due to nonresonant photoconductivity. They are also seen with the other wavelengths and their field position appears independent of the laser wavelength. The origin of these structures is still unknown at present.

We have also measured the $\mathrm{SdH}$ oscillations of the dc resistance ${ }^{7} \rho_{x x}$ and found that the resonance magnetic field for $\lambda=96 \mu \mathrm{m}$ is close to a minimum in $\rho_{x x}$ (i.e., the Fermi level is between two Landau subbands) and that for $\lambda=70.6$ $\mu \mathrm{m}$ is close to a $\rho_{x x}$ maximum (i.e., the Fermi level is close to the middle of a Landau subband). In the $\lambda=96 \mu \mathrm{m}$ case, the CR signal is about five times the strongest nonresonant signal, which is the low field shoulder of the resonance in Fig. 1. At $\lambda=70.6 \mu \mathrm{m}$, on the other hand, both components of the signal are about equal in magnitude.

Two effects have to be considered to explain the physical origins of the observed resonance in full detail. The first is heating of the sample at the resonance conditions, in which case the signal simply reflects the temperature dependence of the resistance. Qualitatively, the signal at $\lambda=96 \mu \mathrm{m}$ can be explained by this mechanism. However, the sample is in liquid helium and more quantitative account cannot be made. We know from transmission experiments on the same sample that the absorption at $\mathrm{CR}$ is strong, the relative change in transmission being $\Delta T / T \sim 20 \%$, corresponding to $\sim 2 \mu \mathrm{W}$ absorbed power in the sample. The second is that of a change of the resistance due to the redistribution of carriers between Landau levels. In a 2D system, the resistance $\rho_{x x}$ vanishes almost completely when the Fermi level lies between two Landau levels. ${ }^{8}$ In such a case one Landau level is completely filled and the next higher one, separated by several kT in energy, is completely empty. The vanishing of $\rho_{x x}$ is due to the fact that no scattering is allowed in this situation. The presence of FIR radiation changes this situation drastically because both the photoexcited carriers in the higher Landau level and the empty states left behind in the lower Landau level increase scattering and a strong increase of $\rho_{x x}$ is expected. When the Fermi level is in the middle of a Landau level, which is partially empty, a much weaker relative change of the resistance may be expected. Both mechanisms, heating and carrier redistribution, qualitatively describe the magnitude and the sign of the photoconductive signal. The observed long relaxation time may suggest a thermal mechanism, but this observation alone is insufficient to exclude the redistribution mechanism.

In conclusion, it may be stated that cyclotron resonance of 2D electrons in GaAs-AlGaAs heterojunctions can be easily observed in photoconductivity. Due to the high sample quality which can be obtained with this system, the observed signal is sufficiently strong $(\Delta R / R=0.5 \%$ for 100 $\mu \mathrm{W} / \mathrm{cm}^{2}$ intensity), that the effect may be useful as a narrow band tunable detector. The linewidth of $0.3 \mathrm{~T}$ corresponds to a bandwidth of $1.2 \times 10^{11} \mathrm{~Hz}$ and resolution $r / \Delta r$ of 10 is already attained at $3 \mathrm{~T}$. More detailed investigations are needed to make it into a practical detector, to clarify the mechanism for the observed photoconductivity, and to elucidate the physics which is responsible for the steps and the plateaus in the resistance as observed in the Shubnikov-de Haas effect. The selective redistribution of carriers between different Landau levels, as can be realized by cyclotron resonant absorption, should provide another method to investigate carrier localization in $2 \mathrm{D}$ systems.

${ }^{1}$ Y. Katayama, N. Kotera, and K. F. Komatsubara, in Proc. of the 2nd Conf. on solid state Devices, Tokyo 1970; J. Jpn. Soc. Appl. Phys. 40, Suppl. 214 (1971).

${ }^{2}$ C. C. Hu, J. Pearse, K. M. Chan, and R. G. Wheeler, Surf. Sci. 73, 207 (1978), and the references therein.

${ }^{3}$ A. Darr, A. Huber, and J. P. Kotthaus, Proceedings of the III International Conference of Physics of Narrow Gap Semiconductors (PWN Polish Scientific, Warsaw, 1978), p. 418.

${ }^{4}$ C. F. Lavine, N. J. Wagner, and D. C. Tsui, in Proc. 4th Int. Conf. on Electronic Properties of 2D Systems, New London, 1981; Surf. Sci. (to be published).

${ }^{5}$ Y. Shiraki, J. Phys. C 10, 4549 (1977).

${ }^{6}$ R. Dingle, H. L. Stormer, A. C. Gossard, and W. Wiegmann, Appl. Phys. Lett. 33, 665 (1978).

${ }^{7}$ D. C. Tsui and A. C. Gossard, Appl. Phys. Lett. 38, 550 (1981).

${ }^{8}$ D. C. Tsui, H. L. Stormer, and A. C. Gossard, Phys. Rev. B 25, 1405 (1982). 